\title{
System tests of the LHCb RICH detectors in a charged particle beam
}

\author{
Hugh Skottowe ${ }^{\dagger}$, University of Cambridge \\ on behalf of the LHCb RICH collaboration
}

\begin{abstract}
The RICH detectors of the LHCb experiment will provide efficient particle identification over the momentum range $1-100 \mathrm{GeV} / \mathrm{c}$. Results are presented from a beam test of the LHCb RICH system using final production pixel Hybrid Photon Detectors, the final readout electronics and an adapted version of $\mathrm{LHCb}$ RICH reconstruction software. Measurements of the photon yields and Cherenkov angle resolutions for both nitrogen and $\mathrm{C}_{4} \mathrm{~F}_{10}$ radiators agree well with full simulations. The quality of the data and the results obtained demonstrate that all aspects meet the stringent physics requirements of the experiment are now ready for first data.
\end{abstract}

\section{INTRODUCTION}

LHCb [1] is one of the four major experiments of the Large Hadron Collider at CERN. It is a single forward-arm spectrometer which will study $\mathrm{CP}$ violating effects and rare decays of $\mathrm{B}$ mesons to high precision. The physics aims of the experiment require efficient hadron identification which is provided by two separate Ring Imaging Cherenkov (RICH) detectors.

Three different radiator media are used in the two RICH detectors to cover a wide momentum range [2]. RICH1 is located directly after the precision vertex detector and before the magnet and uses Silica Aerogel $(1<p<10 \mathrm{GeV} / c)$ and $\mathrm{C}_{4} \mathrm{~F}_{10}(10<p<60 \mathrm{GeV} / c)$ radiators. The $\mathrm{RICH} 2$ detector is located between the tracking sub-detectors and the calorimeters and uses $\mathrm{CF}_{4}$ to cover the range $16<p<100 \mathrm{GeV} / c$.

The Hybrid Photon Detectors (HPDs) consist of a pixelated silicon detector anode which is encapsulated in a vacuum envelope. The vacuum tube has a quartz entrance window with a photocathode deposited on the inside. Photoelectrons are accelerated towards the silicon pixel detector using a $20 \mathrm{kV}$ cross-focusing electron optics system.

Previous beam-tests [4] using pre-production HPDs and data-aquisition electronics successfully demonstrated that the individual components meet or exceed the stringent requirements of the $\mathrm{LHCb}$ experiment. The system test presented here uses HPDs and on-detector Level-0 electronics [5] from the final production, and pre-production prototypes of the off-detector Level-1 [6] electronics boards. The test provides an important validation of the final $\mathrm{RICH}$ architecture in an environment as close to the later operation in the $\mathrm{LHCb}$ experiment as possible.

The tests were performed in late 2006 with a beam of $80 \mathrm{GeV} / c$ pions from the CERN SPS producing saturated Cherenkov rings. The SPS was in a special configuration

$\dagger$ Email: skottowe@hep.phy.cam.ac.uk

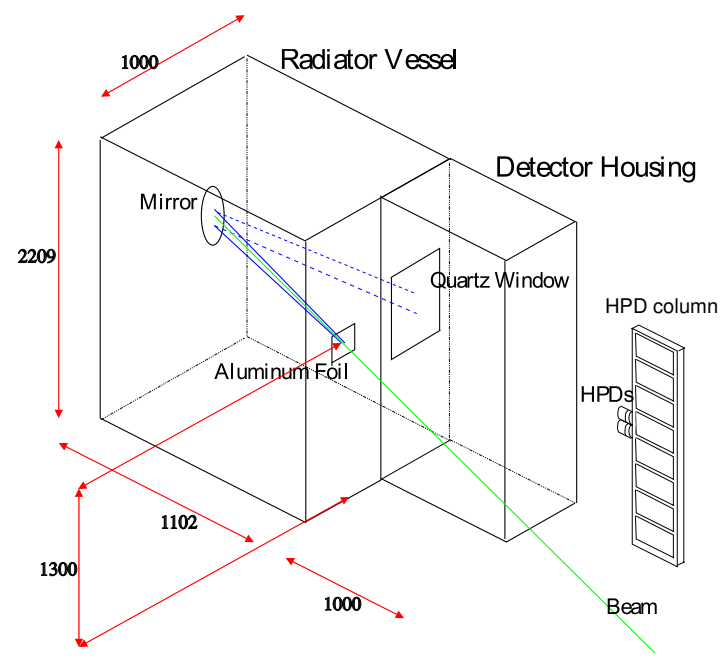

Fig. 1. Schematic diagram of experimental setup for system tests. The beam from the CERN SPS enters the radiator volume through a foil window. Cherenkov photons emitted by the charged particles of the beam are reflected by an adjustable mirror onto a plane of HPDs in the detector volume.

with $25 \mathrm{~ns}$ bunch spacing, specially adapted to emulate the LHC crossing frequency. Both $\mathrm{N}_{2}$ and $\mathrm{C}_{4} \mathrm{~F}_{10}$ were used as a Cherenkov radiator. The use of $\mathrm{C}_{4} \mathrm{~F}_{10}$ has the advantage that this gas is also used in the RICH1 sub-detector of the $\mathrm{LHCb}$ experiment and results from the beam-tests can directly be translated to the final detector.

A total of 36 final production HPDs was arranged on three columns, using the final support structure, but with a single customized radiator vessel and mirror configuration (see figure 1). This allowed a Cherenkov ring to be projected onto a single HPD when using $\mathrm{N}_{2}$ radiator, whereas the Cherenkov ring covers up to four HPDs when using $\mathrm{C}_{4} \mathrm{~F}_{10}$ radiator, due to the higher refractive index.

The position and direction of the beam particles were recorded with two silicon pixel detectors of the same type as used in the HPDs and with the charged beam passing directly through each sensor. The data from the beam position monitor could then be read out through the same data-acquisition electronics and $\mathrm{LHCb}$ event builder as the data obtained from the HPDs.

Data recorded during the system tests was analysed using the full LHCb reconstruction and analysis software with minimal modifications to take the customized setup of the test into account. Simulated events were obtained using the $\mathrm{LHCb}$ simulation and digitization software. 

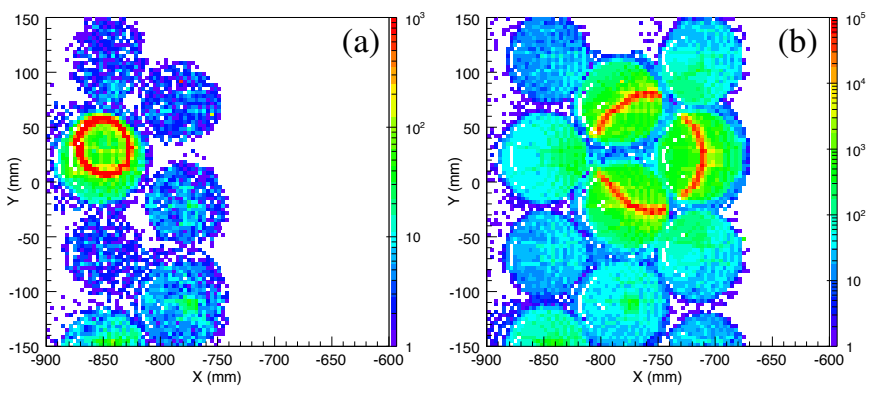

Fig. 2. Accumulated Cherenkov ring distributions before alignments and corrections (a) using Nitrogen gas as Cherenkov radiator with the ring image contained within a single HPD and (b) using $\mathrm{C}_{4} \mathrm{~F}_{10}$ gas as Cherenkov radiator with the ring image covering three HPDs.

Figure 2 shows hits accumulated in HPDs over many events before adjustments and alignments to the data. Clear Cherenkov rings are visible, both contained in a single HPD in the case of $\mathrm{N}_{2}$ radiator, and over three HPDs in the case of $\mathrm{C}_{4} \mathrm{~F}_{10}$ radiator. There is good separation between the signal and background, with much higher hit density on the ring than in the surrounding area.

\section{Alignment}

The beam tests were used as a test of the procedure for aligning the LHCb RICH optical setup for the purposes of reconstructing the data. It has been shown that it is possible to determine mirror misalignments using recorded Cherenkov data by considering the sinusoidal variation of the Cherenkov angle, $\theta_{\mathrm{C}}$, with respect to the angle around the ring, $\phi$, that results from such misalignment. Once the mirror alignment parameters have been determined in this way, they can be used for further reconstruction studies.

In the case of $\mathrm{C}_{4} \mathrm{~F}_{10}$ radiator, where the Cherenkov ring is imaged on three or four HPDs, the variation in $\theta_{\mathrm{C}}$ with respect to $\phi$ is also dependent on other alignment parameters: the alignment and rotation of individual HPDs. Consequently, these parameters must be adjusted so as to make the $\theta_{\mathrm{C}}$ distribution uniform within the $\phi$ range corresponding to individual HPDs, as shown in figure 3.

\section{CHARGE SHARING}

The signal produced by the primary photoelectron in an HPD is usually contained within a single pixel of the silicon chip but it is possible for a signal to be over threshold in two or more adjacent pixels, resulting in two or more recorded hits.

Data were recorded using a set of red LEDs in order to measure the probability of this charge sharing effect. The charge sharing probability was then determined by counting the number of adjacent two-pixel clusters, taking into account a correction due to the probability of two genuine photoelectrons striking adjacent pixels. The systematic uncertainty was then evaluated by considering contributions from background sources such as ion feedback and field settling effects, as well
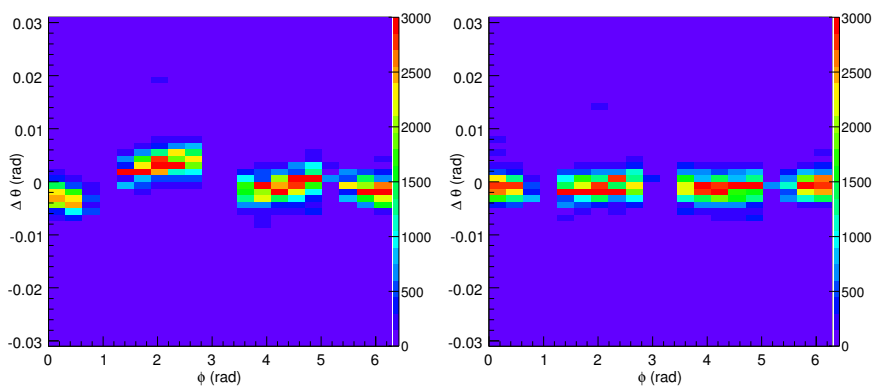

Fig. 3. Difference between the measured and expected Cherenkov angle $\left(\Delta \theta_{\mathrm{C}}\right)$ as a function of the angle around the Cherenkov ring, $\phi$, with $\mathrm{C}_{4} \mathrm{~F}_{10}$ radiator, after preliminary mirror alignment (left) and full alignment (right).

\begin{tabular}{c|c} 
HPD No. & charge sharing fraction \\
\hline 117 & $(2.8 \pm 0.0$ (stat.) \pm 0.2 (syst.)) $\%$ \\
222 & $(4.1 \pm 0.0$ (stat.) \pm 2.0 (syst.) $\%$ \\
265 & $(1.7 \pm 0.1$ (stat.) \pm 1.1 (syst.) $\%$ \\
282 & $(3.1 \pm 0.1$ (stat.) \pm 0.3 (syst.) $\%$
\end{tabular}

TABLE I

CHARGE SHARING FRACTIONS MEASURED FROM LED DATA INCLUDING STATISTICAL AND SYSTEMATIC UNCERTAINTIES.

as variation of the nominal size of the photocathode image on the silicon sensor. The results for the HPDs used in the determination of the photon yield are summarized in table I.

\section{Photoelectron Yield}

One of the key measures of the efficiency of a RICH detector is the average number of photons detected per incident charged particle. In the case of LHCb RICH and HPDs, several factors must be considered in the determination of this photoelectron yield. Ion feedback, caused by photoelectrons scattering from atoms inside the HPD vacuum tube, and field settling effects are concentrated respectively at the centre and edge of the photocathode image on the pixel chip. In order to remove these effects from the analysis, an annulus region is defined by the expected Cherenkov ring position and size variation, and events are only included in the analysis if at least four hits were recorded within the annulus region, and no more than three hits outside.

The beam used for the tests contained a mixture of particles: approximately $80 \% \pi^{-}, 10 \%$ electrons, $7 \%$ kaons and $3 \%$ anti-protons with an estimated uncertainty of $1 \%$ [7]. The photoelectron yield is extracted by performing a constrained fit to the distribution of number of hit pixels per event, using a Poisson distribution for each single particle contribution, and for a two particle contribution (where both particles are assumed to be pions). In the case of $\mathrm{C}_{4} \mathrm{~F}_{10}$, a three particle contribution was also included, and the beam composition was not used in the fit, as the higher refractive index prevents the different particle species contributions from being disentangled. The values of the contributions from charge sharing and, in the case of $\mathrm{N}_{2}$, double-hits and beam-composition, were fixed to the measured values in the fit and varied within their uncertainties to evaluate the size of the systematic uncertainties. 


\begin{tabular}{l|c|c|c} 
HPD & Measured & Expected & Ratio \\
\hline 117 & $12.44 \pm 0.04_{-0.09}^{+0.08}$ & $12.15 \pm 0.61$ & $1.02 \pm 0.05$ \\
264 & $13.08 \pm 0.03_{-0.12}^{+0.15}$ & $13.98 \pm 0.70$ & $0.94 \pm 0.05$ \\
265 & $12.76^{-0.12} \pm 0.03_{-0.13}^{+0.16}$ & $12.72 \pm 0.64$ & $1.01 \pm 0.05$
\end{tabular}

TABLE II

MEASURED AND EXPECTED PHOTOELECTRON YIELDS IN $\mathrm{N}_{2}$ AT T $=295 \mathrm{~K}$ AND $\mathrm{P}=9.6 \times 10^{4} \mathrm{~Pa}$.

An example distribution of the number of hit pixels per event with its corresponding fit for an $\mathrm{N}_{2}$ run can be seen in figure 4 (left). The model accurately reproduces the distribution seen in the data. The measured photon yields are compared to the expected yields for each of the HPDs, including the quantum efficiencies measured by the manufacturer, in table II. The expected yields contain an additional HPD efficiency factor which mainly results from Rutherford backscattering at the surface of the silicon anode which reduces the energy of the primary cluster. This efficiency has been determined to be $\approx 85 \%$ [8]. The largest contributions to the uncertainty on the expected yield originate from a $5 \%$ error on the product of the measured quantum efficiency of the HPDs, the transmission of the quartz window separating the gas radiator from the HPD volume and the reflectivity of the mirror. The quoted uncertainty in table II also includes a small contribution from atmospheric pressure and temperature variations during the beam-test period.

In the case of the $\mathrm{C}_{4} \mathrm{~F}_{10}$ radiator, the Cherenkov rings are distributed over three or four HPDs. Each photo-detector therefore accepts a different portion of the ring and we therefore normalize the number of photoelectrons seen in each photo-detector to the azimuthal angle fraction $\Delta \phi$, as measured from the centre of the ring, covered by the active part of each photocathode.

Due to an imperfect gas circulation system and limited time, data taken with $\mathrm{C}_{4} \mathrm{~F}_{10}$ have an a priori unknown concentration of $\mathrm{C}_{4} \mathrm{~F}_{10}$ in the gas radiator volume for each run. Figure 4 (right) illustrates the result of the fit to the photon yield for one run with $90.2 \% \mathrm{C}_{4} \mathrm{~F}_{10}$ purity and table III summarizes the results obtained for two runs of slightly differing $\mathrm{C}_{4} \mathrm{~F}_{10}$ fractions. The quoted $\mathrm{C}_{4} \mathrm{~F}_{10}$ molar fraction is extrapolated from the measured mean Cherenkov angle $\left\langle\theta_{\mathrm{C}}\right\rangle$.

The systematic uncertainty on the photoelectron yield in $\mathrm{C}_{4} \mathrm{~F}_{10}$ has been evaluated considering various possible sources. Doubling the width of the annulus from three to six pixels which is equivalent to doubling the fraction of the HPD area where the hits are counted as signal hits, results in $\sim 3 \%$ higher single particle Poisson mean. This indicates that most signal hits are already included in the default selection and hits from background sources contribute at most at the 3\% level. The measurement of the fraction of azimuthal angle along the Cherenkov ring covered by the HPD has an uncertainty of about $5 \%$. Relaxing or tightening the fit range and leaving the charge sharing fraction as a free parameter rather than fixing it to the measured value contributes only $1 \%$ to the systematic uncertainty. Overall, the systematic uncertainty in the photon yield per radian for $\mathrm{C}_{4} \mathrm{~F}_{10}$ is estimated at $5 \%$.

\begin{tabular}{c|l|c|c|c} 
& HPD & Measured & Expected & Ratio \\
\hline $90.2 \% \mathrm{C}_{4} \mathrm{~F}_{10}$ & 222 & $8.9 \pm 0.5$ & $9.3 \pm 0.5$ & $1.0 \pm 0.1$ \\
$\mathrm{~T}=296.5 \mathrm{~K}$ & 265 & $8.6 \pm 0.5$ & $8.9 \pm 0.5$ & $1.0 \pm 0.1$ \\
$\mathrm{P}=9.59 \times 10^{4} \mathrm{~Pa}$ & 283 & $8.7 \pm 0.5$ & $9.3 \pm 0.5$ & $0.9 \pm 0.1$ \\
\hline $94.7 \% \mathrm{C}_{4} \mathrm{~F}_{10}$ & 265 & $8.9 \pm 0.5$ & $9.3 \pm 0.5$ & $1.0 \pm 0.1$ \\
$\mathrm{~T}=297.3 \mathrm{~K}$ & 282 & $9.3 \pm 0.6$ & $9.3 \pm 0.6$ & $1.0 \pm 0.1$ \\
$\mathrm{P}=9.60 \times 10^{4} \mathrm{~Pa}$ & 283 & $9.1 \pm 0.5$ & $9.6 \pm 0.5$ & $1.0 \pm 0.1$
\end{tabular}

TABLE III

MEASURED AND EXPECTED PHOTOELECTRON YIELDS IN $\mathrm{C}_{4} \mathrm{~F}_{10}$ PER UNIT $\Delta \phi$. THE QUOTED UNCERTAINTIES CONTAIN BOTH STATISTICAL AND SYSTEMATIC CONTRIBUTIONS ADDED IN QUADRATURE, AND ARE DOMINATED BY THE SYSTEMATIC CONTRIBUTION.
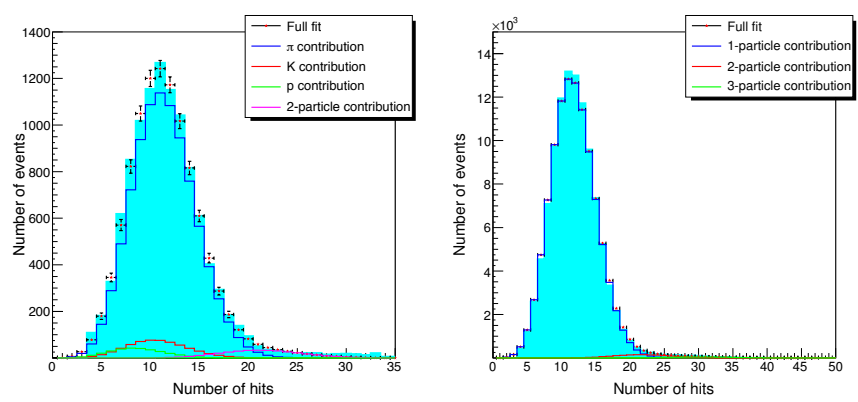

Fig. 4. The observed number of Cherenkov photons per event (points) and fit (line) measured using $\mathrm{N}_{2}$ (left) and $\mathrm{C}_{4} \mathrm{~F}_{10}$ (right) radiators.

\section{Cherenkov Angle ReCOnstruction}

The resolution of the reconstructed Cherenkov angle is one of the key parameters in the evaluation of the performance of the LHCb RICH detectors. Details of the reconstruction algorithm can be found in [9]. The Cherenkov angle, $\theta_{\mathrm{C}}$, is reconstructed for each photon hit and is measured with respect to the centre of the ring. The ring centre is measured from the known spatial parameters of the charged particle when imaged onto the photo-detector plane.

The distributions of $\theta_{C}$ per event for $\mathrm{N}_{2}$ and $\mathrm{C}_{4} \mathrm{~F}_{10}$ radiators are shown in figure 5. The distributions shown are for data and simulated events. The distributions are obtained by taking the weighted mean of the individual hit measurements associated to a given particle for both data and simulation. Figure 5 (left) shows the distribution obtained for data taken with the $\mathrm{N}_{2}$ radiator and figure 5 (right) shows the corresponding distribution for $\mathrm{C}_{4} \mathrm{~F}_{10}$. The simulated hits for $\mathrm{N}_{2}$ agree well with the distribution obtained from data and illustrate the high level of precision which we can expect from the RICH detectors of the LHCb experiment. Due to the imperfect knowledge of the exact fraction of $\mathrm{C}_{4} \mathrm{~F}_{10}$ in the gas admixture, the Sellmeier coefficients (which determine the gas refractive index) were tuned so that the mean Cherenkov angle in the simulated events agreed with the mean angle measured in data. Whilst the absolute measurement of the Cherenkov angle for pure $\mathrm{C}_{4} \mathrm{~F}_{10}$ was therefore not possible, the Sellmeier tuning method allowed an accurate comparison between data and simulation of the Cherenkov angle resolution. Assuming a 90/10 admixture of $\mathrm{C}_{4} \mathrm{~F}_{10} / \mathrm{N}_{2}$ in the simulation gave a residual shift of $0.65 \mathrm{mrad}$ in the mean of the simulated distribution compared to data. This was attributed to imperfect knowledge 

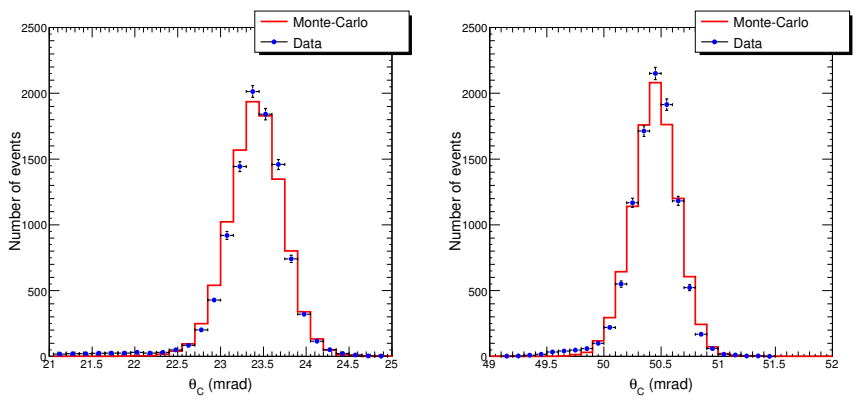

\section{REFERENCES}

[1] The LHCb Collaboration, JINST 3 (2008) S08005

[2] LHCb RICH Technical Design Report, CERN/LHCC 2000-037, CERN/LHCC 2003-059

[3] M. Moritz et al., IEEE Trans. Nucl. Sci. 51 (2004) 1060

[4] M. Adinolfi et al., Nucl. Instr. and Meth. A 574 (2007) 39

[5] M. Adinolfi et al., Nucl. Instr. and Meth. A 572 (2007) 689

[6] C. P. Barham et al., to be submitted to JINST

[7] H. Atherton et al., CERN Yellow Report 80-07 (1980)

[8] N. Kanaya et al., Nucl. Instr. and Meth. A 553 (2005) 41

[9] R. Forty [LHCb Collaboration], Nucl. Instr. and Meth. A 433 (1999) 257

Fig. 5. Cherenkov angle resolution for real (points) and simulated data (line), with $\mathrm{N}_{2}$ radiator (left) and $\mathrm{C}_{4} \mathrm{~F}_{10}$ radiator (right).

of the actual $\mathrm{C}_{4} \mathrm{~F}_{10}$ fraction, as well as temperature and atmospheric pressure effects. This residual shift was then corrected for by fine-tuning in the simulated events.

In order to extract the Cherenkov angle resolution, each $\theta_{\mathrm{C}}$ distribution is described by a double-Gaussian with common mean. The Cherenkov angle resolution is taken as the width of the core distribution. For the case of $\mathrm{N}_{2}$, the resolution is measured as $\sigma_{\theta}=(0.296 \pm 0.003$ (stat.)) $\mathrm{mrad}$ and agrees well with the value obtained for simulated events $\sigma_{\theta}=(0.28 \pm 0.01$ (stat.)) mrad. The resolution in events recorded with $\mathrm{C}_{4} \mathrm{~F}_{10}$ as radiator medium is determined as $\sigma_{\theta}=(0.166 \pm 0.002$ (stat.)) mrad which agrees well with the value obtained for simulated events, $\sigma_{\theta}=(0.158 \pm 0.001$ (stat.)) mrad. The additional tails in the data compared to the simulation are due to residual background effects such as electronic noise and reflection of Cherenkov photons off the HPD mechanical structure which are not yet modelled in the full $\mathrm{LHCb}$ simulation software.

\section{CONCLUSION}

The results obtained in this test beam provide a major step towards the realization of the LHCb RICH detectors. For the first time, the final production hardware and software have been utilized to produce the first results from an $\mathrm{LHCb} \mathrm{RICH}$ detector in a realistic configuration. The results with $\mathrm{C}_{4} \mathrm{~F}_{10}$ radiator are the first such studies with Cherenkov rings over several HPDs and an important step towards understanding data from the full $\mathrm{LHCb}$ RICH.

Using a beam matching the $25 \mathrm{~ns}$ structure of the LHC, it has been successfully demonstrated that all aspects of the $\mathrm{RICH}$ operation meet the stringent requirements of the $\mathrm{LHCb}$ experiment. The data have been recorded using the $\mathrm{LHCb}$ data-acquisition software and the results obtained using the full $\mathrm{LHCb}$ simulation and reconstruction framework. Both the measured Cherenkov angle resolution and the photoelectron yield confirm the results of previous beam-tests [4] and agree with simulation. The RICH detectors have now been commissioned, and have recorded data during the first circulation of LHC beams. The detectors will be ready to take data when the LHC beams start colliding in early 2009 and, based on the results of these tests, we expect excellent performance. 